\title{
Effects of core stability exercise combined with virtual reality in collegiate athletes with nonspecific low back pain: a randomized clinical trial
}

\author{
Osama Ragaa Abdelraouf ${ }^{1}$, Amr Almaz Abdel-aziem¹, Ali Osman Selim² and Olfat Ibrahim Ali ${ }^{3^{*}}$ [D
}

\begin{abstract}
Background: Nonspecific low back pain (LBP) in collegiate athletes is shown to be accompanied by poor core endurance. Consequently, trunk muscle stabilization exercises for lumbar instability are widely used. Virtual reality (VR) training can activate the cerebral cortex and enhance the cortex to control balance and improve motion function. The purpose of the study was to assess the effect of combined core stability exercises (CSE) and VR training in improving body balance and function in collegiate male athletes with nonspecific low back pain (LBP).

Results: The post-values of the experimental group were significantly higher than that of the control group for the dynamic balance in anterior $(p=0.031)$, posterolateral $(p=0.034)$, and posteromedial $(p=0.037)$ directions. Moreover, there was a significant difference in the post-values of both groups regarding reducing the Micheli Functional Scale in favor of the experimental group ( $p=0.012)$.

Conclusions: CSE training plus virtual reality is more effective than CSE training alone in improving total body balance and dysfunction level in collegiate male athletes with nonspecific LBP. It is recommended that clinicians consider CSE combined with VR to maximize the improvement in overall body balance when developing rehabilitation programs for collegiate athletes with nonspecific low back pain.

Trial registration: Pan African Clinical Trial Registry, PACTR201907749053096. Retrospectively registered on 15 April 2019. https://pactr.samrc.ac.za/Researcher/ManageTrials.aspx
\end{abstract}

Keywords: Balance, Collegiate athletes, Core stability exercise, Low back pain, Virtual reality

\section{Background}

Nonspecific low back pain (LBP) is identified as low back pain which is not due to a known specific pathology. Pain is located below the costal margin and above the inferior gluteal folds, with or without leg pain [1]. It is the most costly among athletic populations [2].

The lumbar segment instability and decreased muscle control are important factors in LBP. Consequently,

\footnotetext{
*Correspondence: Olfat_ib@yahoo.com; Olfat_ib@cu.edu.eg ${ }^{3}$ Department of Basic Science for Physical Therapy, Faculty of Physical Therapy, Cairo University, 7 Ahmed Elziat Street, Ben Elsaryat, El Dokki, Giza 12611, Egypt

Full list of author information is available at the end of the article
}

trunk muscle stabilization exercises for lumbar instability are widely used in a clinical setting [3]. Spinal stability is obtained from simultaneous activities of the trunk muscles, and active simultaneous contractions are necessitated to improve the spinal stability of LBP patients with unstable lumbar segments [4].

Strengthening exercises of the abdominal, trunk, and pelvic floor muscles are required to improve LBP [5]. Core stability exercises (CSE) have become one of the fitness trends that broadly used exercises for LBP. It improves athletic performance, prevents injuries, and alleviates LBP [6]. 
There is evidence of increased fatigue, decreased cross-section, and fatty infiltration of the paraspinal muscles in patients with nonspecific LBP [7]. So, prolonged exercise programs targeting the trunk muscles in patients with nonspecific LBP have shown to increase the cross-sectional area of the muscle which plays a major role in spinal function [8].

Virtual reality (VR) technology is being used for its capability benefit as a therapeutic intervention for restoring coordinated movement patterns. VR training can activate the cerebral cortex and enhance the cortex to control balance and improve motion function [9]. This technology provides the most appropriate, individualized motor learning pattern. Similarly to computer games, VR rehabilitation exercises are important in terms of patient motivation [10].

Bezerra et al. [11] concluded that virtual environment considers a promising tool for the assessment and during the training of healthy subjects. Also, Trost et al. [12] found that the VR technology has been effectively applied to acute pain treatment, and recent reviews have suggested their potential usefulness in chronic pain. It is reported that the virtual walking combined with physiotherapy decrease pain and kinesiophobia and enhance function in patients with LBP [1].

The previous studies either investigated the effect CSE on the muscle activity, balance [13], and spinal proprioception [14] or examined the effect of CSE combined with traditional physiotherapy for patients with neurological impairments [15] or CLBP [16].

Recently, nonspecific LBP in collegiate athletes is shown to be accompanied by poor core endurance. So, there is a lack of experimental works that examined the effect of CSE combined with VR on balance and spinal dysfunction in athletes with nonspecific LBP [17].

Therefore, the purpose of this trial was to investigate the effect of CSE training combined with VR on balance and function in collegiate male athletes with nonspecific LBP. We hypothesized that the addition of VR to CSE training would improve the body balance and the dysfunction level in young athletes with nonspecific LBP compared to CSE training alone.

\section{Methods}

\section{Participants}

Fifty male collegiate athletes (football players) aged ranging from 18 to 24 years old, who presented with nonspecific LBP, were recruited for this single-blind, randomized clinical trial in which participants were randomized to one of two treatment groups: experimental group performed CSE while wearing Oculus Rift as a VR system, and control group performed CSE without the VR system. The sample size was calculated was using the G"Power 3.1.9.2 software (Universities, Dusseldorf,
Germany). The effect size (ES) was 0.9 based on the $p$ value (0.05) and power of the test $(0.80)$.

All participants signed an informed consent and agreed with the study in advance. The study was performed in accordance with the Declaration of Helsinki and approved by the local institutional review board (Approval No. P.T.REC/012/002039). The demographic data of the participants are illustrated in Table 1.

Participants with the following inclusion criteria were included in the current study: (1) orthopedist-confirmed diagnosis of nonspecific LBP (2) having 2 or more episodes of LBP during the last year [17], (3) positive prone instability test was eligible [18] and the prone instability test has a sensitivity of 0.72 and is used to identify individuals who exhibit lumbar segmental instability with poor muscular control [19], and (4) they were not engaged in any treatment program during the study. Patients with nerve root manifestations; underlying systematic or visceral disease; conditions such as ankylosing spondylitis, spondylolysis, and spondylolisthesis; previous low back surgery [20]; vestibular dysfunctions; and lower extremity injury within the past month or concussion within the past 3 months were excluded [21].

Recruitment took place in two steps: firstly, an orthopedic specialist from the university hospital identified the potentially eligible athletes and referred them to the Faculty of Physical Therapy Biomechanics Lab. Secondly, the researcher conducted another screening for criteria of patient's selection to make the final decision regarding eligibility. $\mathrm{Pa}$ tients who meet the eligibility criteria were given oral and written information explaining the procedure and importance of the study, and those who decided to contribute to the study were assigned to one of the treatment groups.

Randomization was conducted using a computergenerated random table, and opaque sealed envelopes were used for the group's allocation which is prepared according to the patients' total number. Randomization and allocation were prepared by a statistician who was not involved in the study before the beginning of the study to ensure concealment of patient allocation.

The participants' flow diagram throughout the different steps of the trial is shown in Fig. 1.

\section{Procedure}

Star excursion balance test (SEBT) was utilized for dynamic balance evaluation as the primary experimental outcome. SEBT was accomplished while participants were standing on the dominant leg, and participants were asked to reach as far as they could along a grid in the anterior, posteromedial, and posterolateral directions with the tip of the great toe of the non-stance limb. Participant's hands were held at the iliac crest during the test. Six practices followed by 2 -min rest and three test trials were performed in each direction. The reaching 
Table 1 Demographic data of participants

\begin{tabular}{llll}
\hline & Experimental group, $n=25$ & Control group, $n=25$ & $p$ value \\
\hline Age, years & $20.86 \pm 5.17$ & $22.14 \pm 2.58$ & 0.075 \\
Height, $\mathrm{cm}$ & $173.44 \pm 4.26$ & $175.32 \pm 4.30$ & 0.127 \\
Weight, $\mathrm{kg}$ & $70.76 \pm 4.43$ & $73.16 \pm 6.04$ & 0.116 \\
BMl, $\mathrm{kg} / \mathrm{m}^{2}$ & $23.56 \pm 1.79$ & $23.85 \pm 2.40$ & 0.626 \\
\hline
\end{tabular}

Data are presented as mean \pm standard deviation; $p<0.05$ means significant difference

$p$ value probability level, $n$ numbar

distance of the grid was read and recorded manually, and the average of the three test trials was calculated.

The order of reaching directions was randomized. The test was rejected and then repeated if a participant failed to maintain a unilateral stance, lifted or moved the standing foot from the grid, or failed to return the reaching limb to the starting position. For the assurance of accurate analysis, the data of the reaching distance was normalized by leg length to exclude the impact of the leg length. The leg length was measured from the anterior superior iliac spine most distal end to the most distal end of the lateral malleolus on each limb [22]. SEBT reliability ranged from 0.89 to 0.94 . Reaching in the anterior, posteromedial, and posterolateral directions has excellent reliability. For the normalized maximum excursion distances, the intraclass correlation coefficients $(1,1)$ ranged from 0.86 to 0.92 [23]. SEBT was performed before and after 6 weeks of intervention.
The Micheli Functional Scale (MFS) was used to assess the level of dysfunction of athletes and pain intensity of low back pain. The MFS is a 5-item questionnaire; its components include a symptom question, 3 activityrelated questions (extension, flexion, and jumping), and a visual analog scale (VAS) for pain intensity. The questionnaire is constructed to assess back pain symptoms and smoothness or difficulty of performing different sporting activities related to low back pain. Responses were calculated as follows: symptom question response $(0-5$ points) and the 3 activity questions (extension, $0-4$; flexion, $0-3$; jumping, $0-3$ ) with a total of 15 points. The visual analog scale is scored on 10 points on a $10-\mathrm{cm}$ line. The global score is calculated by adding MFS questionnaire responses and VAS scores. This summation of the questioner equals a maximum score of 25 [24].

This number is then multiplied by 4 with the total scores ranging from 0 to 100 . The lower the score, the less

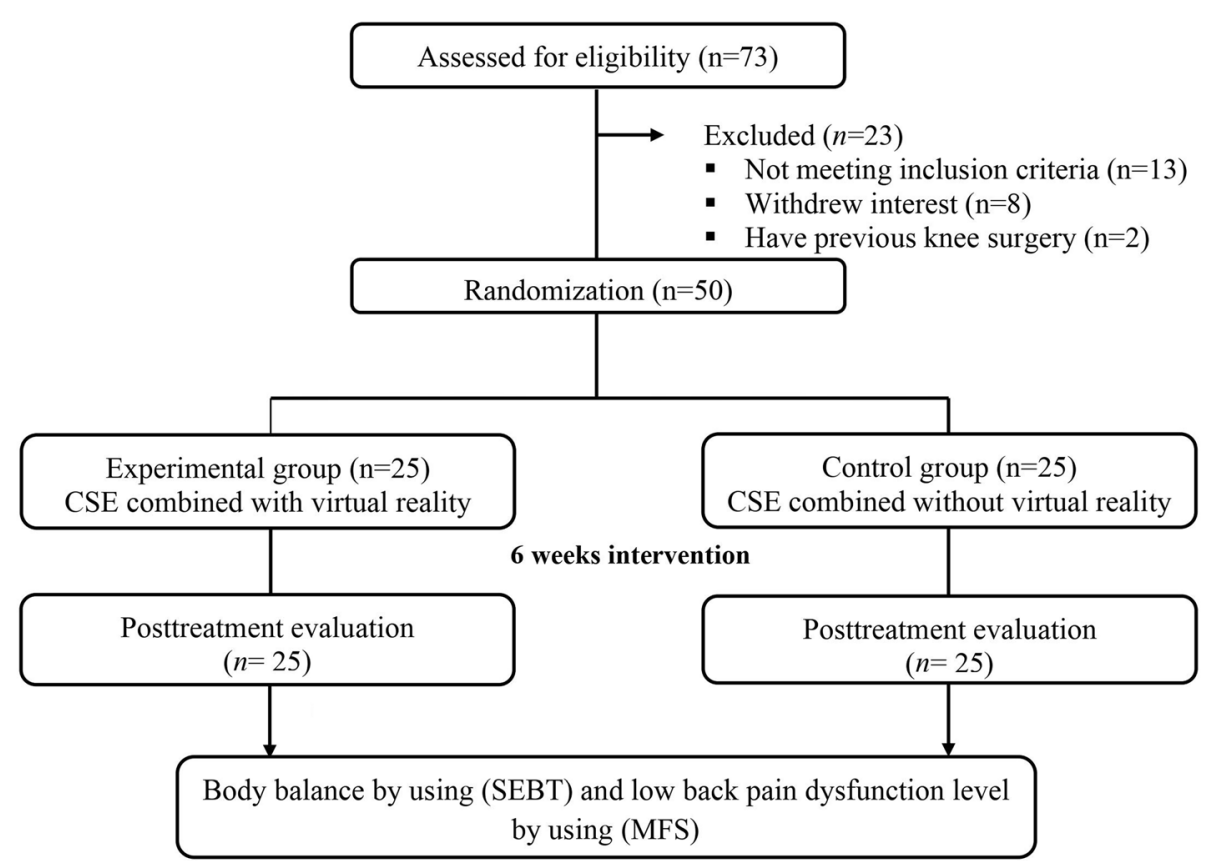

Fig. 1 Flow diagram of participant progress through the study. CSE, core stability exercises; SEBT, star excursion balance test; MFS, Micheli Functional Scale 
dysfunction, as 0 scores indicate the least amount of dysfunction and 100 indicates the worse dysfunction [24].

The validity and reliability of the MFS questionnaire were settled, and its reliability was excellent $(\alpha=0.904)$. There is a positive high correlation between MFS scores and modified Oswestry LBP Disability Questionnaire scores $(r=0.90)$ [24].

The control group was instructed to perform three CSE 5 days/week over a period of 6 weeks under the supervision of a certified physical therapist in the Biomechanics Laboratory. The CSE was conducted for 20 repetitions with $15 \mathrm{~s}$ hold for each exercise. The whole exercise set was repeated twice with 3-min rest in between [25].

For the prone plank, the subjects assumed a front support position resting on the forearms with shoulders directly over their elbows and legs are straight out behind. Hips were lifted to a degree which makes the whole body from the shoulders to the ankles leveled and supported only on forearms and toes. This position could be maintained through the coactivation of both lower abdominal and back muscles. For the side plank, the subjects lay on each side as they balance on their forearms with the shoulder directly above the elbow and legs are straight. The pelvis was lifted so that the whole body was balanced on the forearm and feet. The body formed a straight line, and the oblique muscles should be felt down the side of the trunk working to maintain the position. For the Superman plank, subjects laid on their stomach with arms straight over the head. Both arms and legs were lifted off the ground and kept in this position for the holding period [14] (Fig. 2).

The experimental group performed the same CSE program of the control group while wearing Oculus Rift DK2 as a means for VR (Fig. 2). The Oculus Rift is a lightweight headset, which is developed by Oculus VR (Facebook Technologies LLC, CA, USA) to allow its user to feel like they are actually in a game and could look at any direction. The screen displays two images adjacent to each other, one for each eye. The combination of lenses is placed above the screen, enabling the zoom in-out and reshaping the picture for both eyes, thereby creating stereoscopic 3D images. The image was adjusted according to the wearer's head motions which were detected by the embedded sensors in the Rift devices [26]. Two games were selected, one for each exercise set: NoLimits 2 Roller Coaster and Euro Truck 2 simulations. In addition to being enjoyable for most of the participants, both games require frequent head motions and quick direction changes which facilitate activation of the core muscles. A supervised familiarization session was allowed before the start of the 6-week intervention period.

\section{Statistical analysis}

The data were found to be normally distributed as indicated by normal histogram curves. Moreover, Shapiro-Wilk's normality test was non-significant $(p>0.05)$. In addition, the results of Levene's test revealed that there were no significant differences between the variances of the tested groups of the

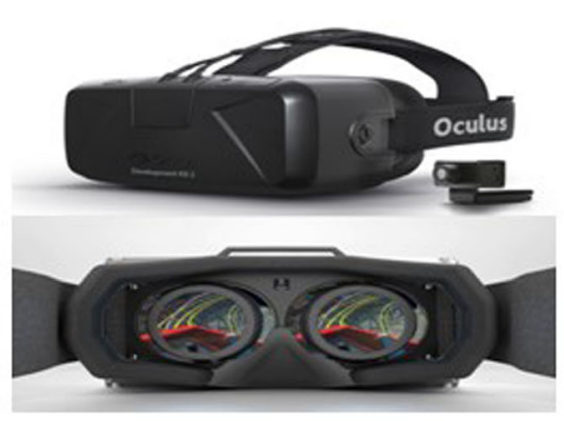

2-A

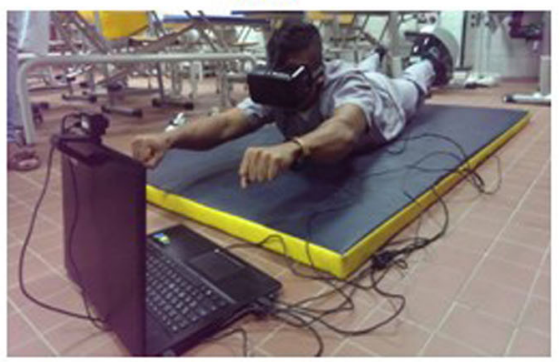

2-C

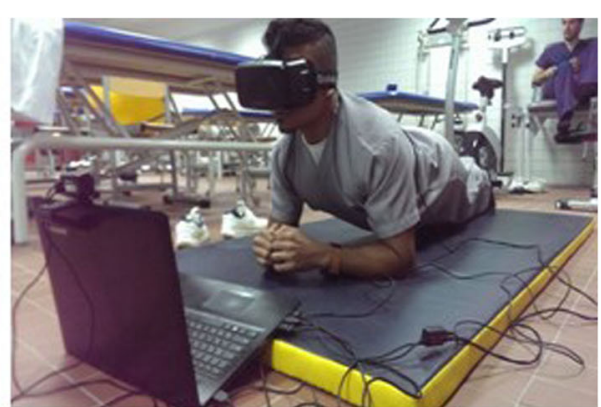

2-B

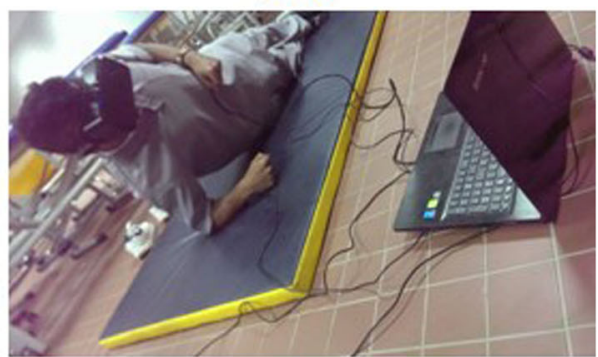

2-D

Fig. 2 Core stability exercises. a The Oculus Rift DK2 headset. b The prone plank with Oculus. c Superman plank with Oculus. d The side plank with Oculus 
tested dependent variables $(p>0.05)$. Based on the previous findings of the normality and homogeneity assumptions, the parametric analysis was conducted.

Data were analyzed using SPSS version 20 (Armonk, NY: IBM Corp.). Unpaired $t$ test was used to compare demographic variables of participants. Multi-way analysis of variance (MANOVA) was used to compare balance and Micheli Functional Scale of both groups, with time as the within-participant factor (baseline measurement vs. post-intervention measurement) and the group as the between-participant factor (experimental vs. control). In case the $F$ ratio was significant, the differences between means were examined using the Tukey test. Unpaired $t$ test was used to compare the change score of the tested variable between the groups. The level of significance was set at 0.05 for all tests.

\section{Results}

There was no significant difference between both groups in age, weight, height, and BMI $(p>0.05)$. Descriptive statistics of the pre- and post-intervention values of balance and Micheli Functional Scale of the two groups is illustrated in Table 2.

There was no significant difference between the preintervention values of SEBT of both groups in the anterior, posterolateral, and posteromedial directions $(p=$ $0.386,0.828,0.843$, respectively). The post-intervention values of both groups were significantly higher than preintervention values in the three directions $(p=0.001)$. The post-intervention values of SEBT of the experimental group were significantly higher than the postintervention values of the control group in the three directions ( $p=0.031,0.034,0.037$, respectively).

There was no significant difference between the preintervention values of MFS of both groups $(p=0839)$. Both groups' post-intervention values were significantly lower than the pre-intervention values $(p=0.001)$. The experimental group's post-intervention value of MFS was significantly lower than the control group's postintervention value $(p=0.012)$.

Analysis of the change scores shown that the experimental group had significantly more changes in anterior, posterolateral, and posteromedial directions of SEBT and MFS $(p=0.001)$ compared with the control group, as shown in Table 2 .

\section{Discussion}

The hypothesis of the current study was confirmed by the results which revealed that CSE using a VR system is more effective in improving balance, reducing the spinal dysfunction than using CSE alone in athletes with nonspecific LBP. The CSE is known to strengthen the deep core muscles of the human body, such as the local spinal muscles, the abdominal muscles, the hip muscles, and the pelvic muscles, which generate all the power and motility of the human body. In the athletes, strengthening these core muscles would improve spinal movement and stability and greatly help to improve athletic performance [27].

The improvement of balance and spinal functions in both groups can be explained by the effect of CSE that work to improve the coordination and control of the trunk muscles and harmonize the physiological functions by stimulating proprioception, which ultimately improves balance sense, and postural maintenance ability [28]. It has been demonstrated that the CSE is effective in not only rehabilitating and preventing the LBP [15], but also for improving balance [27]. However, Aggarwal et al. [28] found that balance training, as well as CSE training, leads to enhancement in both static and dynamic balance in the recreationally active subjects.

The significant improvement in the three tested directions of the SEBT for both groups is supported by the finding of Imai et al. [22]. It has been stated that CSE improved both static and dynamic balance immediately [22].

Previous studies also showed that VR could improve significantly pain and movement reproducibility with training which indicates that VR-based programs could produce significant proprioceptive and functional improvements $[16,29]$.

Moreover, the positive effect of the CSE on pain intensity might be understood by the recent findings of Paungmali et al. [30] who concluded that the CSE could possibly influence the $\beta$-endorphin but not the plasma cortisol level among nonspecific chronic LBP patients.

Table 2 The values of balance (cm) and Micheli Functional Scale (out of 100)

\begin{tabular}{|c|c|c|c|c|c|c|}
\hline & \multicolumn{3}{|c|}{ Experimental group, $n=25$} & \multicolumn{3}{|c|}{ Control group, $n=25$} \\
\hline & Pre-value & Post-value & Change score & Pre-value & Post-value & Change score \\
\hline Balance (anterior) & $91.28 \pm 18.05$ & $102.64 \pm 18.34^{*}$ & $11.36 \pm 2.36$ & $90.04 \pm 13.98$ & $92.40 \pm 12.90^{* \dagger}$ & $2.36 \pm 3.03$ \\
\hline Balance (posterolateral) & $87.12 \pm 13.75$ & $96.08 \pm 12.82^{*}$ & $8.96 \pm 2.05$ & $86.24 \pm 14.73$ & $87.44 \pm 15.05^{* \dagger}$ & $1.20 \pm 1.04$ \\
\hline Balance (posteromedial) & $84.24 \pm 13.76$ & $94.40 \pm 12.95^{*}$ & $10.16 \pm 1.43$ & $83.44 \pm 14.15$ & $86.40 \pm 13.39^{* \dagger}$ & $2.96 \pm 1.88$ \\
\hline Micheli Functional Scale & $47.16 \pm 6.04$ & $39.28 \pm 5.98^{*}$ & $-7.88 \pm 2.22$ & $46.84 \pm 5.01$ & $43.44 \pm 5.24^{* \dagger}$ & $-3.40 \pm 1.26$ \\
\hline
\end{tabular}

Data are presented as mean \pm standard deviation

*Significant difference between pre- and post-values $(p<0.05)$

${ }^{\dagger}$ Significant difference between post-values $(p<0.05)$

$n$ numbar 
The mechanism of action of CSF for pain-relieving might be attributed to an endogenous opioid mechanism and not involved a stress-induced analgesia mechanism.

The superior results obtained by combining CSE and VR in this study could be understood by the fact that the simulation of a real environment is increasingly utilized to restore motor function and enhance balance dysfunction [31]. Bolton et al. reported that the balance control depends on the central nervous system at multiple levels [32]; the current finding was supported by Mao et al. [10] as they have shown that the VR environment activates the cortical and sub-cortical areas, thus enabling the cortex to improve balance and motion function [10]. Moreover, Basso Moro et al. [33] revealed that, when healthy individuals performed an incremental swing balance task in a semi-VR environment, the oxygenation increase in the prefrontal cortex of both hemispheres.

Mao et al. [10] reported that VR technology has unique advantages in the rehabilitation field compared to conventional therapies. First, treatment is more interesting for patients, and the sports favored by the patients in daily life can be utilized as a training program. Second, patients can perform usual training in any position on a wheelchair or sitting as well as standing or walking; there is no need for stabilized posture control. Third, the game system is inexpensive and easy to carry and operated at any place

Moreover, compared with proprioceptive neuromuscular facilitation training, VR training stimulates the body and not only the involved limbs by enriching proprioceptive information inputs [10]. The VR balance training could induce more reasonable visual and proprioceptive input and enhance postural stability, the reaction time, balance, and walking function [32]. Therefore, VR balance games can be used as a potent and useful tool to train adults with balance dysfunction [34]. In the same context, Yilmaz Yelvar et al. [13] stated that integration of virtual walking with the traditional physiotherapy reduces pain and kinesiophobia and improved function in patients with sub-acute and chronic nonspecific LBP which concurs with the current findings. VR-guided exercises may specifically enhance exercise effectiveness in the younger population [35].

Kim et al. [36] studied the effects of a virtual realitybased yoga program on the adult female with LBP, and they found a positive effect on pain intensity and spinal stability. Despite the difference in the sample criteria, their results support the current findings. However, Eser et al.'s [37] finding did not come along with the present results as they failed to find a statistically significant difference between VR therapy and conventional therapy in lower extremity motor recovery and mobility training in stroke patients. This may explain the differences in the reported results as they were working on neurologically impaired subjects
Transmission of motor task training to the real or virtual environment is not yet fully determined. Todorov et al.'s [38] results showed that subjects who underwent the virtual environment training performed the task much better than participants who practice the same amount of training in a real environment. In contrast, another study assessed VR exercise and real-world exercise in a pick-and-place task and revealed improvement in both groups, but those who exercised in the real world task improved more [39].

Ther are some limitations present in this trial. Firstly, this trial was delimited to male collegiate athletes, thus limiting the generalizability of the results. Secondly, the VR system that has been used is still in development. It is recommended to conduct a further study using different balance tests in bigger and different populations, including women and older adults in order to generalize the study findings. Moreover, further studies are needed to determine the effect of the addition of the VR system to CSE on the electromyography activities of the back and abdominal muscles.

\section{Conclusion}

This study showed significant improvement in the overall body balance, pain intensity, and spinal dysfunction when the CSE is conducted in combination with VR compared to CSE alone. Therefore, it is recommended that clinicians consider CSE combined with VR to maximize the improvement in the overall body balance when developing rehabilitation programs for collegiate athletes with nonspecific low back pain.

\section{Abbreviations \\ BMI: Body mass index; CSE: Core stability exercises; ES: Effect size; LBP: Low back pain; MANOVA: Multi-way analysis of variance; MFS: Micheli Functional Scale; NLBP: Nonspecific low back pain; SEBT: Star excursion balance test; VAS: Visual analog scale; VR: Virtual reality \\ Acknowledgements \\ The authors wish to thank the participants for their contribution to this study.}

Authors' contributions

All authors have read and approved the final manuscript.

Funding

Self-funding. No funding was received.

Availability of data and materials

Not applicable

Ethics approval and consent to participate

All participants signed an informed consent and agreed with the study in advance. The study was performed in accordance with the Declaration of Helsinki.

Faculty of Physical Therapy Local Institutional Review Board (Approval No. P.T.REC/012/002039)

Consent for publication

Not applicable 


\section{Competing interests}

The authors declare that they have no competing interests.

\section{Author details}

'Department of Biomechanics, Faculty of Physical Therapy, Cairo University, Giza, Egypt. ${ }^{2}$ Department of Physical Therapy for Surgery, Faculty of Physical Therapy, Cairo University, Giza, Egypt. ${ }^{3}$ Department of Basic Science for Physical Therapy, Faculty of Physical Therapy, Cairo University, 7 Ahmed Elziat Street, Ben Elsaryat, El Dokki, Giza 12611, Egypt.

Received: 11 May 2020 Accepted: 3 June 2020

Published online: 09 July 2020

\section{References}

1. Burton AK, Balague F, Cardon G, Eriksen HR, Henrotin Y. Lahad A, Chapter 2. European guidelines for prevention in low back pain: November 2004. Eur Spine J. 2006;15(Suppl 2):S136-8.

2. Bono CM. Low-back pain in athletes. J Bone Joint Surg Am. 2004; 86- A(2): 382-396.

3. Marshall PW, Murphy BA. Core stability exercises on and off a Swiss ball. Arch Phys Med Rehabil. 2005;86:242-9.

4. Kriese M, Clijsen R, Taeymans J, Cabri J. Segmental stabilization in low back pain: a systematic review. Sportverletz Sportschaden. 2010;24:17-25.

5. Ferreira PH, Ferreira ML, Maher CG, Herbert RD, Refshauge K. Specific stabilization exercise for spinal and pelvic pain. A systemic review. Aust J Physiother. 2006:29:79-86.

6. Hodges PW, Richardson CA. Insufficient muscular stabilization of the lumbar spine associated with low back pain. Spine. 1996;24:2435-48.

7. Wan Q, Lin C, Li X, Zeng W, MA C. MRI assessment of paraspinal muscles in patients with acute and chronic unilateral low back pain. Br J Radiol. 2015. 88:20140546. https://doi.org/10.1259/bjr.20140546.

8. Kliziene I, Sipaviciene S, Klizas S, Imbrasiene D. Effects of core stability exercises on multifidus muscles in healthy women and women with chronic low-back pain. J Back Musculoskeletal Rehabil. 2015;28:841-7. https://doi.org/10.3233/BMR-150596.

9. Popescu VG, Burdea GC, Bouzit M, Hentz VR. A virtual reality-based telerehabilitation system with force feedback. IEEE Trans Inf Technol Biomed. 2000;4(1):45-51.

10. Mao Y, Chen $P$, Li L, Huang D. Virtual reality training improves balance function. Neural Regen Res. 2014;9:1628-34.

11. Bezerra IMP, Crocetta TB, Massetti T, Silva TD, Guarnieri R, Meira CM Jr, et al. Functional performance comparison between real and virtual tasks in older adults: a cross-sectional study. Medicine (Baltimore). 2018;97(4):e9612. https://doi.org/10.1097/MD.0000000000009612.

12. Trost Z, Zielke M, Guck A, Nowlin L, Zakhidov D, France CR, et al. The promise and challenge of virtual gaming technologies for chronic pain: the case of graded exposure for low back pain. Pain Manag. 2015;5(3):197-206. https://doi.org/10.2217/pmt.15.6.

13. Yilmaz Yelvar GD, Cirak Y, Dalkilinc M, Parlak Demir Y, Guner Z, Boydak A. Is physiotherapy integrated virtual walking effective on pain, function, and kinesiophobia in patients with non-specific low-back pain? Randomised controlled trial. Eur Spine J. 2017;26(2):538-45. https://doi.org/10.1007/ s00586-016-4892-7.

14. Selkow NM, Eck MR, Rivas S. Transversus abdominis activation and timing improves following core stability training: a randomized trial. Int J Sports Phys Ther. 2017;12(7):1048-56.

15. Karatas M, Cetin N, Bayramoglu M, Dilek A. Trunk muscle strength in relation to balance and functional disability in unihemispheric stroke patients. Am J Phys Med Rehabil. 2004;83:81-7.

16. Monteiro-Junior RS, de Souza CP, Lattari E, Rocha NB, Mura G, Mach-ado S, da Silva EB. Wii-workouts on chronic pain, physical capabilities and mood of older women: a randomized controlled double blind trial. CNS \& Neurological Disorders-Drug Targets (Formerly Current Drug Targets-CNS \& Neurological Disorders). 2015;14(9):1157-64.

17. Abdelraouf OR, Abdel-aziem AA. The relationship between core endurance and back dysfunction in collegiate male athletes with and without nonspecific low back pain. Int J Sports Phys Ther. 2016;11:337-44.

18. Etemadi Y, Salavati M, Arab AM, Ghanavati T. Balance recovery reactions in individuals with recurrent nonspecific low back pain: effect of attention. Gait Posture. 2016:44:123-127. https://doi.org/10.1016/j.gaitpost.2015.11.017.
19. Corkery MB, O'Rourke B, Viola S, Yen S, Rigby J, Singer K. An exploratory examination of the association between altered lumbar motor control, joint mobility, and low back pain in athletes. Asian J Sports Med. 2014;5:4. https://doi.org/10.5812/asjsm.24283.

20. Tsao H, Druitt TR, Schollum TM, Hodges PW. Motor training of the lumbar paraspinal muscles induces immediate changes in motor coordination in patients with recurrent low back pain. J Pain. 2010;11(11):1120-8.

21. Ebadi S, Ansari NN, Naghdi S, Jalaei S, Sadat M, Bagheri $H$. The effect of continuous ultrasound on chronic nonspecific low back pain: a single blind placebo controlled randomized trial. BMC Musculoskelet Disord. 2012;13: 192-201.

22. Imai A, Kaneoka K, Okubo Y, Shiraki H. Effects of two types of trunk exercises on balance and athletic performance in youth soccer players. Int J Sports Phys Ther. 2014;9:47-57.

23. Gribble PA, Kelly SE, Refshauge KM, Hiller CE. Interrater reliability of the star excursion balance test. J Athl Train. 2013;48(5):621-6.

24. d'Hemecourt PA, Zurakowski D, d'Hemecourt CA, Curtis C, Ugrinow V, Deriu L. Validation of a new instrument for evaluating low back pain in the young athlete. Clin J Sport Med. 2012;22:244-8.

25. Barr KP, Griggs M, Cadby T. Lumbar stabilization: a review of core concepts and current literature. Am J Phys Med Rehabil. 2007;86:72-80

26. Yoon S, Sung D, Park GD. The effect of active core exercise on fitness and foot pressure in Taekwondo club students. J Phys Ther Sci. 2015;27:509-11.

27. Goradia I, Doshi J, Kurup L. A review paper on Oculus Rift and Project Morpheus. Inter J Current Eng Technology. 2014;4(5):3196-200.

28. Aggarwal A, Zutshi K, Munjal J, Kumar S, and Sharma V. Comparing stabilization training with balance training in recreationally active individuals. Inter J Ther Rehabil. 2010;17:244-53.

29. Alemanno F, Houdayer E, Emedoli D, Locatelli M, Mortini P, Mandelli C, et al Efficacy of virtual reality to reduce chronic low back pain: proof-of-concept of a non-pharmacological approach on pain, quality of life, neuropsychological and functional outcome. PLoS One. 2019;14(5):e0216858 https://doi.org/10.1371/journal.pone.0216858.

30. Paungmali A, Joseph LH, Punturee $K$, et al. Immediate effects of core stabilization exercise on $\beta$-endorphin and cortisol levels among patients with chronic nonspecific low back pain: a randomized crossover design. J Manipulative Physiol Ther. 2018:41(3):181-8.

31. McEwen D, Taillon-Hobson A, Bilodeau M, Sveistrup $H$, and Finestone $H$. Virtual reality exercise improves mobility after stroke: an inpatient randomized controlled trial. Stroke. 2014;45:1853-5.

32. Bolton DA, Brown KE, Mcllroy WE, Staines WR. Transient inhibition of the dorsolateral prefrontal cortex disrupts somatosensory modulation during standing balance as measured by electroencephalography. Neuroreport. 2012:23:369-72.

33. Basso Moro S, Bisconti S, Muthalib M, Spzialetti M, Cutini S, Ferrari M. A semi-immersive virtual reality incremental swing balance task activates prefrontal cortex: a functional near-infrared spectroscopy study. Neuroimage. 2014:85:451-60.

34. Singh DK, Rajaratnam BS, Palaniswamy V, Pearson H, Raman VP and Bong PS. Participating in a virtual reality balance exercise program can reduce risk and fear of falls. Maturitas. 2012;73:239-43.

35. Schwesig R, Goldich Y, Hahn A. Postural control in subjects with visual impairment. Eur J Ophthalmol. 2011;21:303-9.

36. Kim SS, Min WK, Kim JH, Lee BH. The effects of VR-based Wii fit yoga on physical function in middle-aged female LBP patients. J Phys Ther Sci. 2014; 26(4):549-52. https://doi.org/10.1589/jpts.26.549.

37. Eser F, Yavuzer G, Karakus D, karaoglan B. The effect of balance training on motor recovery and ambulation after stroke: a randomized controlled trial. Eur J Phys Rehabil Med. 2008:44:19-25.

38. Todorov E, Shadmehr R, Bizzi E. Augmented feedback presented in a virtual environment accelerates learning of a difficult motor task. J Mot Behav. 1997;29(2):147-58

39. Bryanton C, Bosse J, Brien M, et al. Feasibility, motivation, and selective motor control virtual reality compared to conventional home exercise in children with cerebral palsy. CyberPsychol Behav. 2006;9:123-8.

\section{Publisher's Note}

Springer Nature remains neutral with regard to jurisdictional claims in published maps and institutional affiliations. 